\title{
Maternal Sleep and Fetal Outcome
}

\author{
Riva Tauman*
}

Dana Children's Hospital, Tel Aviv Medical Center, Tel Aviv University, Tel Aviv 64239, Israel

\begin{abstract}
Pregnancy is associated with profound changes in sleep. Up to $75 \%$ of pregnant women experience some form of sleep disruption during pregnancy. The emerging data from recent years suggests that sleep disturbances may represent a novel contributor to adverse pregnancy outcomes and that sleep disorders during pregnancy may affect both maternal and fetal outcomes. In spite of the increased awareness, information specifically addressing sleep disturbances and fetal outcomes is limited. The current review summarizes the existing published literature on the effect of short sleep duration, poor quality sleep and sleep-disordered breathing (SDB) on the developing fetus.
\end{abstract}

Keywords: Pregnancy, sleep, fetus, infant, sleep-disordered breathing, short sleep duration.

\section{INTRODUCTION}

The importance of good quality sleep and the negative impact of sleep disturbances are well established in the general population and many publications exist on the associations between sleep and a wide spectrum of medical conditions. In particular, sleep disordered breathing (SDB), poor sleep quality and short sleep duration have all been linked to the development of the metabolic syndrome, type 2 diabetes mellitus, cardiovascular morbidity and mortality [1-7].

Pregnancy is a time of great change in a woman's life and is associated with profound changes in sleep. Up to $75 \%$ of pregnant women experience some form of sleep disruption during pregnancy [8-10]. The emerging data from recent years suggests that sleep disturbances may represent a novel contributor to adverse pregnancy outcomes and that sleep disorders during pregnancy may affect both maternal and fetal outcomes. In spite of the increased awareness, information specifically addressing sleep disturbances and fetal outcomes is limited. The current review evaluates/examines the existing published literature on the effect of short sleep duration, poor quality sleep and SDB on the developing fetus.

\section{POOR SLEEP AND SHORT SLEEP DURATION DURING PREGNANCY AND FETAL OUTCOME}

Short sleep duration has become a major public health concern in the past several decades in western countries. Self reported sleep duration of Americans has decreased by 1.5-2 hours over the past 40 years and more than $30 \%$ of adults between the ages of 30 and 64 years report sleeping less than 6 hours per night [11]. Accumulating data indicate that chronic sleep restriction is associated with decreased learning and cognitive functioning, accidents and errors in workplace, alterations in fat and glucose metabolism, increased risk of obesity and increased mortality [1-3, 12].

*Address correspondence to this author at the Dana Children's Hospital, Tel Aviv Medical Center, Tel Aviv University, Tel Aviv 64239, Israel; Tel: 972-3-6974614; 972-524262025, Fax: 972-3-6974634;

E-mail: tauman@tasmc.health.gov.il
During pregnancy and postpartum period, women are at particular risk for sleep restriction. The optimal duration of sleep in pregnancy is unknown. However, descriptive studies of sleep duration throughout pregnancy have found an increase in total sleep time and daytime sleepiness during the first trimester and a decrease in sleep time in the second and third trimesters $[9,13,14]$. The changes in sleep needs and sleep duration during pregnancy are most likely due to the hormonal, physical and emotional changes of pregnancy. These changes are also responsible for the increase in sleep complaints such as backaches, urinary frequency, restless legs, leg cramps, fetal movements, heartburn, and general discomfort throughout the pregnancy period [15]. All of the above result in profound changes in sleep quality even in the absence of sleep curtailment.

To date, there is only a small number of studies that examined the effect of short sleep duration and poor sleep on fetal outcome.

Prematurity is a major public health issue and a leading cause of infant morbidity and mortality. Although some maternal and fetal risk factors for preterm deliveries have been identified, nearly $50 \%$ of preterm births are due to unknown causes. In a recent study from Greece, using a large mother-child cohort, Micheli and colleagues have found that women with sleep deprivation $(<5$ hours of sleep at the third trimester) were at high risk for preterm births (RR 1.7; 1.1-2.8), with highest risk observed for medically indicated preterm births; i.e programmed cesarean section or induced vaginal birth (RR 2.4; 1.0-6.4) after adjusting for maternal age, education, parity, smoking during pregnancy , and pre-pregnancy BMI [16]. In a longitudinal study on 166 women, using the Pittsburgh Sleep Quality Index, Okun and colleagues have shown that poor sleep quality was a predictor of preterm birth, with the largest effects in early pregnancy (14-16 weeks) and more modest effects in later pregnancy (30-32 weeks). With every one-point increase on the PSQI, the odds of preterm birth increase $25 \%$ in early pregnancy and $18 \%$ in later pregnancy [17].Currently, the mechanisms underlying these associations remain unclear and need to be elucidated/clarified. However, poor sleep may contribute to increased risk for preterm birth both 
independently, as well as in conjunction with other established risk factors, such as stress. In other words, low sleep quality may be a marker of psychosocial stress which is a known risk factor for preterm births.

The length and mode of delivery are two factors that can affect fetal outcome and the newborn's wellbeing. Few studies have thus far examined the effect of maternal sleep duration and sleep quality on labor and delivery process. In a prospective observational study, using actigraphy as an objective measure of sleep, Lee and colleagues have shown that women who slept less than 6 hours of sleep per night during the last month of pregnancy had a significantly longer mean duration of labor and a higher rate of cesarean sections than women getting more than 6 hours of sleep [18]. Correlation between sleep duration and length of delivery stages was also reported in another cross sectional study [19]. Using the Pittsburgh Sleep Quality questionnaire, Naghi and colleagues examined the relationship between sleep quality and type and duration of labor. They found that poor sleepers (PSQI $>5$ ) were $20 \%$ more likely to undergo cesarean section and had longer labor duration [20]. In a cross sectional study on 457 pregnant women with singleton pregnancy and gestational age greater than 37 weeks and after excluding hypertension, gestational diabetes mellitus (GDM) or emergency cesarean section, Zafarghandi and colleagues have found that sleep duration and quality can affect the type of delivery and length of labor stages. In addition, reported sleep duration of more than 8 hours was associated with higher rates of high Apgar scores (Apgar score greater than 9) compared with reported sleep duration of less than 8 hours ( $86 \%$ vs. $92 \%, p=0.001)$. No significant differences were found in birth weight [19].

\section{MATERNAL SLEEP DISORDERED BREATHING (SDB) AND FETAL OUTCOMES}

SDB is a common sleep disorder among pregnant women. The term SDB refers to the spectrum of breathing disorders during sleep, ranging from uncomplicated snoring to the most severe form of SDB, obstructive sleep apnea (OSA). These breathing disorders are characterized by repeated episodes of partial or complete upper airway obstruction during sleep and can result in disruption of normal ventilation, intermittent hypoxia, and arousals from sleep [21]. Physiologic and hormonal changes that occur during pregnancy including gestational weight gain, pregnancy-associated nasopharyngeal edema and decreased functional residual capacity predispose women to upper airway narrowing and the development of SDB [22]. Indeed, habitual snoring has been reported in up to $46 \%$ of pregnant women [23].

Repetitive episodes of obstructive respiratory events during sleep giving rise to cyclic episodes of maternal hypoxemia and repetitive episodes of increased blood pressure and peripheral vasoconstriction may affect placental delivery, fetal growth and fetal well being. Although habitual snoring is common during pregnancy [23], there is a paucity of data regarding the precise incidence and prevalence of OSA in pregnant women. There are also limited data on the association between maternal SDB and fetal outcomes and the limited literature examining such association has produced mixed findings.
In animal models, exposure to intermittent hypoxia during pregnancy has been shown to cause adverse fetal outcomes [24-26]. In two separate studies, Gozal and colleagues have shown that exposure of pregnant rats to brief repetitive episodes of intermittent hypoxia led to fetal growth restriction and impaired ventilatory and resuscitative responses of their offspring to hypoxic and anoxic conditions $[25,26]$.

Several human studies have examined maternal-fetal outcomes in pregnant women with symptoms of SDB, however none of the studies focused solely on fetal outcome.

Using subjective assessment of sleep (questionnaire) Loube and colleagues have studied 350 women and found no significant differences between snorers and non-snorers in regard to neither birth weights nor Apgar scores [27]. In a prospective survey of sleep symptoms in 325 pregnant women, Hedman and colleagues found no differences in birth weight between infants born to snorers and non-snorers [13]. Applying a different methodological approach, Yin and colleagues examined the hypothesis that OSA is more common in pregnancies complicated by hypertensive disease and fetal growth restriction by using nocturnal oxygen saturation monitoring [28]. They found no relation between OSA and hypertensive disease of pregnancy or fetal growth restriction. Similarly, Ayrim and colleagues studied 200 pregnant women and 200 age-matched controls, using a questionnaire that includes questions regarding snoring, witnessed apnea, sleepiness and neck circumference. They found that although the incidence of snoring appears to increase during pregnancy, especially in women who gain excessive weight, there is no effect of fetal outcome [29]. These findings were confirmed by a study on 276 women from Korea [30]. In a recent study from our group on 246 healthy pregnant women with uncomplicated pregnancies, we have shown that maternal snoring is not associated with fetal growth restriction nor with lower Apgar scores [31].

In contrast to the above publications, several studies have demonstrated adverse fetal outcomes in maternal SDB. In a retrospective, cross sectional, consecutive case series from Sweden on 502 women, using a questionnaire about snoring, witnessed apnea and daytime fatigue, Franklin and colleagues have found that self reported habitual snorers developed significantly higher rates of gestational hypertension, preeclampsia and delivery of small for gestational age (SGA) infants than did non-snorers. Differences remained significant after adjustment for age, weight and smoking status [32]. However, the relative contribution of maternal hypertension and preeclampsia to fetal growth and fetal outcomes was not investigated so that it is unknown whether increased risk for these outcomes could be attributed to uncomplicated snoring alone. More recently, using a large mother-child cohort from Greece, Micheli and colleagues have found that women with severe snoring (snored frequently or always during the third trimester) were at high risk for low birth weight; i.e. weight below 2500 grams (relative risk=2.6; [1.2-5.4]) and fetalgrowth restricted neonates, i.e. birth weight below the $10^{\text {th }}$ percentile of the predicted weight distribution $(\mathrm{RR}=2.0$; $[1.0$ 3.9]) after adjusting for maternal age, education, smoking during pregnancy, and pre-pregnancy body mass index [16]. 
In a cross sectional survey on 1000 subjects that were recruited immediately (24-48 hours) postpartum Bourjeily and colleagues evaluated the relationship between SDB symptoms and pregnancy and neonatal outcomes. Using the Multivariable Apnea Prediction Index (MAPI), subjects completed a questionnaire regarding SDB symptoms in the 3 months preceding delivery. It has been shown that symptoms of SDB were associated with higher likelihood of pregnancyinduced hypertension and pre-eclampsia, gestational diabetes and unplanned cesarean deliveries. Maternal gasping was associated with higher likelihood of preterm deliveries, after adjusting for age and multi-fetal pregnancies but this association appeared to be mediated by pre-eclampsia [33]. In a retrospective cohort study of 57 women with a confirmed diagnosis of OSA, Louis and colleagues studied 114 obese women and 114 non-obese controls matched for year of delivery. They found that OSA was associated with an increased risk of preterm delivery, pregnancy complications and maternal morbidity [34]. Among the OSA group, the majority of preterm deliveries were the result of maternal or fetal indication rather than from spontaneous labor or membrane rupture. However, most of the women in the obese control group did not have sleep studies and thus OSA could not be excluded in these patients. In addition, there is no information regarding CPAP compliance in the OSA group, which might have had an impact on the rate of complications if patients were either highly compliant or noncompliant with their therapy.

In a prospective cohort study comparing Berlin Questionnaire scores between pregnant (4074) women at labor and non-pregnant (490) women, Higgins and colleagues compared fetal outcomes such as infant birth weight and Apgar scores. Not surprisingly, women with positive Berlin questionnaire were heavier. Higher incidence of preeclampsia was found in the positive Berlin questionnaire group (OR of 3.0 vs. 1.1). There was no difference in the cumulative percentage of infants with Apgar score $<7$ at 1 - or 5 - minutes born to mothers with positive Berlin compared to those with negative questionnaires, However, in those women with a positive category 2 on the Berlin questionnaire (sleepiness), the percentage of infants with Apgar score $<7$ at 1 - and 5minutes was significantly different [34]. In contrast to other publications in regard to birth weight, the authors found that infant weight was higher in the positive Berlin group [35]. Similar findings were observed by Olivarez and colleagues on a prospective cohort of pregnant women. The authors investigated the relationship of OSA measured by symptombased measures for diagnosis (by the Berlin and Epworth questionnaires) to common perinatal outcomes controlling for obesity in multivariate models. They showed that the majority of OSA cases occur among the obese women and that among non-obese gravidae, the frequency of preeclampsia was significantly higher among women with OSA (adjusted odds ratio $=6.85,95 \%$ confidence interval=1.04,38.5; $\mathrm{p}=0.035$ ). Among the obese women, infant birthweight ratio (birthweight by gestational age) was higher with OSA + screening than OSA- (1.099 vs. 1.035, $\mathrm{p}=0.04)$ and this association remained significant after adjustment for potential confounders $(\mathrm{p}=0.05)$ [36].

Using population-based databases, Chen and colleagues have investigated 791 women who were diagnosed with
OSA within one year prior to their delivery and 3955 agematched controls. The authors performed conditional logistic regression analyses to examine the risks of adverse pregnancy outcomes between the two groups. They found that compared with women without OSA, adjusted odds ratios for low birth weight (LBW), preterm birth, small for gestational age (SGA) infants, cesarean sections, and preeclampsia in women with OSA were 1.76, 2.31, 1.34, 1.74 and 1.6, respectively after adjustment for maternal education, marital status, gestational diabetes, gestational hypertension, anemia, coronary heart disease, hyperlipidemia, obesity, geographic region, parental age, infant's sex and parity [37] They concluded that pregnant women with OSA are at increased risk for having LBW, preterm, and SGA infants, cesarean birth, and preeclampsia compared with pregnant women without OSA. However, this study suffers from several limitations. The major limitation is the absence of information regarding maternal BMI. Obesity is a known risk factor for the development of OSA and for adverse pregnancy outcomes and it is likely that obesity is the underlying mechanism responsible to all the adverse pregnancy outcomes found in this study. Similar to other studies showing adverse maternal and fetal outcomes, it is also possible that some of the adverse pregnancy outcomes (such as preeclampsia) are the mediating mechanism between OSA and adverse fetal outcome and not the OSA per se. In addition the lack of information regarding OSA severity and the percentage of patients treated with continuous positive airway pressure (CPAP) might have also affect the results of the study.

Animal studies and small human case reports have demonstrated that maternal apneic episodes are accompanied by fetal heart rate alterations. These heart rate alterations may be a contributing factor to adverse fetal outcomes. Exposure of rats to chronic or prolonged intermittent maternal hypoxia has shown to cause fetal bradycardia and decreased fetal breathing movements [26]. In one of the first publications on OSA during pregnancy, Joel-Cohen and Schoenfeld reported 3 cases of clinical OSA, not confirmed by polysomnography (PSG), with apnea-associated changes in fetal heart rate [38]. However, a case report of severe OSA during pregnancy revealed normal fetal heart rate reactivity, on cardiotocographic recording (CTG) even during maternal apneic episodes associated with severe desaturations [39].

In a prospective observational study, Sahin and colleagues investigated simultaneously the PSG and nonstress test (NST) records of pregnant women with OSA in order to observe NST changes during maternal desaturation. Four out of 35 women $(11.4 \%)$ had OSA. Of those, 3 had fetal heart decelerations accompanying maternal desaturation. The neonates of women diagnosed with OSA had lower Apgar scores and birth weights compared with neonates of women without OSA [40].

In a larger sample size of 100 women in a prospectively acquired cohort, Olivarez and colleagues investigated the association between sleep-related maternal apnea events and changes in fetal oxygenation status, as measured by fetal heart rate monitoring (FHM). The women underwent at least 3 hours of nocturnal PSG with FHM. Most of the participants were Hispanic and were admitted for unrelated conditions, preterm labor, GDM, pregnancy induced 
hypertension, premature rupture of membranes, chronic hypertension, trauma, and urinary tract infection. Twenty percent were diagnosed with OSA. Among the 20 subjects with OSA the only FHM abnormality that occurred in the study participants was variable decelerations that were of appropriate gestational age. Moreover, none of the apnea episodes were associated with any fetal tracing abnormality. Thus, apnea episodes were not accompanied by any significant changes in the fetal heart rate [36].

While results regarding fetal growth, Apgar score and prematurity are inconclusive, evidence does suggest that maternal SDB bears some risk to the developing fetus. In a recent publication from our group on 222 pregnant women with uncomplicated pregnancy, we found that maternal snoring is associated with enhanced fetal erythropoiesis manifested by increased cord blood levels of nucleated red blood cells, erythropoietin and interleukin-6. These findings provide preliminary evidence that maternal snoring is associated with subtle alterations in markers of fetal wellbeing [41].

In summary, conflicting data exists about the effect of maternal SDB on the fetus. Although some studies report an association with fetal growth, Apgar score and even prematurity, other studies suggest that there is no effect on the fetus. Conflicting data exists also about the effect of maternal apneic episodes on fetal heart rate alterations. Most of the published literature to date is based on subjective assessment of sleep and lacks objective measures that could provide information regarding disease severity. The differences found between studies can be explained by the fact that most of the studies investigated both maternal and fetal outcomes and, therefore, included complicated pregnancies that could by themselves affect fetal growth. For example, pregnancy induced hypertension increases the risk of adverse outcomes such as premature delivery and fetal growth retardation. Moreover, some of the studies did not control for important confounders such as obesity, diabetes, hypertension and other complications of pregnancy. Further studies using objective measures of sleep and controlling for important confounders are required in order to understand whether maternal SDB affects the developing fetus.

\section{MATERNAL SLEEP PRACTICES AND THE RISK OF LATE STILLBIRTH}

In high income countries, more than one in 200 births result in a stillbirth $[42,43]$ which therefore remains an important public health issue, with little change in its rate over the past two decades [43]. In a recent case control study, Stacey and colleagues have investigated the potential impact of SDB and sleep practices on the developing fetus and the risk of stillbirth [44]. They investigated 155 women with singleton late stillbirth ( $>28$ week's gestation) and compared them to 310 matched controls. No relation was found between snoring or daytime sleepiness and risk of late stillbirth. However, women who slept on their back or on the right side on the previous night (before stillbirth or interview) were more likely to experience a late stillbirth compared with women who slept on their left side. Moreover, women who got up to the toilet once or less on the last night were more likely to experience a late stillbirth compared with women who got up more frequently. Women who regularly slept during the day in the previous month were also more likely to experience a late stillbirth than those who did not [44]. Although the findings of this study should be seen as only generating a hypothesis and needing validation, they highlight the importance of the research on maternal sleep and fetal outcome and the potential implications that may be derived from this research.

\section{CONFLICT OF INTEREST}

The authors confirm that this article content has no conflicts of interest.

\section{ACKNOWLEDGEMENT}

This review was supported by the Israel Science Foundation (grant No. 707/12).

\section{REFERENCES}

[1] Tamakoshi A, Ohno Y. Self reported sleep duration as a predictor of all cause mortality: results from the JACC study, Japan. Sleep 2004; 27: 51-4.

[2] Cappuccio FP, D'Elia L, Strazullo P, Miller MA. Quantity and quality of sleep and incidence of type 2 diabetes. Diabetes Care 2010; 33: 414-20.

[3] Patel SR, Ayas NT, Malhotra MR, et al. A prospective study of sleep duration and mortality risk in women. Sleep 2004; 27: 440-4.

[4] Attal P, Chanson P. Endocrine aspects of obestructive sleep apnea. J Clin Endocrinol Metab 2010; 95: 483-95.

[5] Levy P, Tamisier R, Arnaud C, et al. Sleep deprivation, sleep apnea and cardiovascular disoeases. Front Biosci 2012; 4: 2007-21.

[6] Tasali E, Ip MSM. Obstructive sleep apnea and metabolic syndrome. Alterations in glucose metabolism and inflammation. Proc Am Thorac Soc 2008; 5: 207-17.

[7] Pack AI, Gislason T. Obstructive sleep apnea and cardiovascular disease: a perspective and future directions. Prog Cardiovasc Dis 2009; 51: 434-51.

[8] National Sleep Foundation. Summary of findings of the 2007 Sleep in America Poll. US: WBA Market Research 2007.

[9] Lee KA, Zaffke ME, McEnany G. Parity and sleep patterns during and after pregnancy. Obstst Gynecol 2000; 95: 14-8.

[10] Okun ML, Coussons-Read ME. Sleep disruption during pregnancy: how does it influence serum cytokines? J Reprod Immunol 2007; 73: 158-65.

[11] National Center for Health Statistics. QuickStats: percentage of adults who reported an average of less than 6 hours of sleep per 24 hour period, by sex and age group - United States, 1985 and 2004. MMWR Morb Mortal Wkly Rep 2005; 54(37): 933.

[12] Banks S, Dinges DF. Behavioral and physiological consequences of sleep restriction. J Clin Sleep Med 2007; 3: 519-28.

[13] Hedman C, Pohjasvaara T, Tolonen U, Suhonen-Malm AS, Myllyla VV. Effects of pregnancy on mother's sleep. Sleep Med 2002; 3: 37-42.

[14] Mindell JA, Jacobson BJ. Sleep disturbances during pregnancy. J Obstet Gynecol Neonatal Nurs 2000; 29: 590-7.

[15] Pien GW, Schwab RJ. Sleep disorders during pregnancy. Sleep 2004; 27: 1405-17.

[16] Micheli K, Komninos I, Bagkeris E, et al. Sleep patterns in late pregnancy and risk of preterm birth and fetal growth restriction. Epidemiology 2011; 22: 738-44.

[17] Okun ML, Dunkel Schetter C, Glynn LM. Poor sleep quality is associated with preterm birth. Sleep 2011; 34: 1493-8.

[18] Lee KA, Gay CL. Sleep in late pregnancy predicts length of labor and type of delivery. Am J Obstet Gynecol 2004; 191: 2041-6.

[19] Zafarghandi N, Hadavand S, Davati A, Mohseni SM, Kimiaiimoghadam F, Torkestano F. The effects of sleep quality and duration in late pregnancy on labor and fetal outcome. J Matern Fetal Neonatal Med 2012; 25(5): 535-7.

[20] Naghi I, Keypour F, Ahari SB, Tavalai SA, Khak M. Sleep disturbance in late pregnancy and type and duration of labour. J Obstet Gynecol 2011; 31: 489-91. 
[21] AASM Task Force. Sleep-related breathing disorders in adults: recommendations for syndrome definition and measurement techniques in clinical research. Sleep 1999; 22: 667-71.

[22] Bobrowski RA. Pulmonary physiology in pregnancy. Clin Obstet Gynecol 2010; 53: 285-300.

[23] Leung PL, Cheong Hui DS, Leung TN, Yuen PM, Lau TK. Sleep disturbances in Chinese pregnant women. BJOG 2005; 112: 156871.

[24] Schwartz JE, Kovach A, Meyer J, McCOnnell C, Iwamoto HS. Brief, intermittent hypoxia restricts fetal growth in SpragueDawley rats. Biol Neonate 1998; 73: 313-9.

[25] Gozal D, Gozal E, Reeves SR, Lipton AJ. Gasping and autoresuscitation in the developng rat: effect of antecedent intermittent hypoxia. J Appl Physiol 2002; 92: 1141-4.

[26] Gozal D, Reeves SR, Row BW, Neville JJ, Guo SZ, Lipton AJ. Respiratory effects of gestational intermittent hypoxia in the developing rat. Am J Respir Crit Care Med 2003; 167: 1540-7.

[27] Loube MD, Poceta JS, Morales MC, Peacock MMD, Mitler MM. Self reported snoring in pregnancy- association with fetal outcome. Chest 1996; 109: 885-9.

[28] Yin TT, Williams N, Burton C, et al. Hypertension, fetal growth restriction and obstructive sleep apnea in pregnancy. Eur $\mathrm{J}$ Obstet Gynecol Reprod Biol 2008; 141: 35-8.

[29] Ayrim A, Keskin EA, Ozol D, Onaran Y, Yildirim Z, Kafali H. Influence of self reported snoring and witnessed sleep apnea on gestational hypertension and fetal outcome in pregnancy. Arch Gynecol Obstet 2011; 283: 195-9.

[30] Ko HS, Kim MY, Lee J, et al. Obstructive sleep apnea screening and perinatal outcomes in Korean pregnant women. Arch Gynecol Obstet 2013; 287(3): 429-33.

[31] Tauman R, Sivan Y, Katsav S, Greenfeld M, Many A. Maternal snoring is not associated with fetal growth restriction. J Matern Fetal Neonatal Med 2012; 25: 1283-6.

[32] Franklin KA, Holmgren PA, Jonsson F, Poromaa N, Stenlund H, Svanborg E. Snoring, pregnancy-induced hypertension, and growth retardation of the fetus. Chest 2000; 117: 137-41.
[33] Bourjeily G, Chalhoub M, Miller MA. Pregnancy and fetal outcomes of symptoms of sleep disordered breathing. Eur Respir J 2010; 36: 849-55.

[34] Louis JM, Auckley D, Sokol RJ, Mercer BM. Maternal and neonatal morbidities associated with obstructive sleep apnea complicating pregnancy. Am J Obstet Gynecol 2010; 202: 261e1-5.

[35] Higgins N, Leong E, Park CS, Facco FL, McCarthy RJ, Wong CA. The Berlin questionnaire for assessment of sleep disordered breathing risk in parturients and non-pregnant women. Int J Obstet Anesth 2011; 20: 22-5.

[36] Olivarez SA, Maheshwari B, McCarthy M, et al. Prospective trial on obstructive sleep apnea in pregnancy and fetal heart rate monitoring. Am J Obstet Gynecol 2010; 202: 552.e1-7.

[37] Chen YH, Kang JH, Lin CC, Wang IT, Keller JJ, Lin HC. Obstructive sleep apnea and the risk of adverse pregnancy outcomes. Am J Obstet Gynecol 2012; 206: 136e1-5.

[38] Joel Cohen SJ, Schoenfeld A. Fetal response to periodic sleep apnea: a new syndrome in obstetrics. Eur J Obstet Gynecol Reprod Biol 1978; 8: 77-81.

[39] Charbonneau M, Falcone T, Cosio MG, Levy RD. Obstructive sleep apnea during prenancy. Therapy and implications for fetal health. Am Rev Respir Dis 1991; 144: 461-3.

[40] Sahin FK, Koken G, Cosar E, et al. Obstructive sleep apnea in pregnancy and fetal outcome. Int J Gynecol Obstet 2008; 100: 1416.

[41] Tauman R, Many A, Deutsch V, et al. maternal snoring during pregnancy is associated with enhanced fetal erythropoiesis - a preliminary study. Sleep Med 2011; 12: 518-22.

[42] Reddy UM, Laughon SK, Sun L, Troendle J, Willinger M, Zhang J. Prepregnancy risk factors for antepartum stillbirth in the United States. Obstet Gynecol 2010; 116: 1119-26.

[43] Cousens S, Blencowe H, Stanton C, et al. National, regional, and worldwide estimates of stillbirth rates in 2009 with trends since 1995: a systematic analysis. Lancet 2011; 377: 1319-30.

[44] Stacey T, Thompson JM, Mitchell EA, Ekeroma AJ, Zuccollo JM, McCowan LM. Association between maternal sleep practices and risk of late stillbirth: a case-control study. BMJ 2011; 342: 1-6.

\footnotetext{
Received: February 09, 2012

Revised: November 27, 2012
}

Accepted: January 26, 2013

(C) Riva Tauman; Licensee Bentham Open.

This is an open access article licensed under the terms of the Creative Commons Attribution Non-Commercial License (http://creativecommons.org/licenses/bync/3.0/), which permits unrestricted, non-commercial use, distribution and reproduction in any medium, provided the work is properly cited. 